\title{
White matter fiber tract segmentation in DT-MRI using geometric flows
}

\author{
Lisa Jonasson ${ }^{\mathrm{a}, *}$, Xavier Bresson ${ }^{\mathrm{a}}$, Patric Hagmann ${ }^{\mathrm{a}, \mathrm{b}}$, Olivier Cuisenaire ${ }^{\mathrm{a}}$, \\ Reto Meuli ${ }^{\mathrm{b}}$, Jean-Philippe Thiran ${ }^{\mathrm{a}, \mathrm{b}}$ \\ ${ }^{a}$ Signal Processing Institute (ITS), Swiss Federal Institute of Technology, (EPFL), CH-1015 Lausanne, Switzerland \\ ${ }^{\mathrm{b}}$ Department of Diagnostic and Interventional Radiology, Lausanne University Hospital (CHUV), CH-1011 Lausanne, Switzerland
}

Received 21 August 2003; received in revised form 24 April 2004; accepted 13 July 2004

Available online 22 October 2004

\begin{abstract}
In this paper, we present a 3D geometric flow designed to segment the main core of fiber tracts in diffusion tensor magnetic resonance images. The fundamental assumption of our fiber segmentation technique is that adjacent voxels in a tract have similar properties of diffusion. The fiber segmentation is carried out with a front propagation algorithm constructed to fill the whole fiber tract. The front is a $3 \mathrm{D}$ surface that evolves with a propagation speed proportional to a measure indicating the similarity of diffusion between the tensors lying on the surface and their neighbors in the direction of propagation. We use a level set implementation to assure a stable and accurate evolution of the surface and to handle changes of topology of the surface during the evolution process. The fiber tract segmentation method does not need a regularized tensor field since the surface is automatically smoothed as it propagates. The smoothing is done by an intrinsic surface force, based on the minimal principal curvature. This segmentation can be used for obtaining quantitative measures of the diffusion in the fiber tracts and it can also be used for white matter registration and for surgical planning.
\end{abstract}

(C) 2004 Elsevier B.V. All rights reserved.

Keywords: White matter segmentation; DT-MRI; Level sets method; Geometric flows; Fiber tract segmentation

\section{Introduction}

Diffusion tensor magnetic resonance imaging (DTMRI) is a new modality that permits non-invasive quantification of the water diffusion in living tissues. The diffusion tensor (DT) provides information about the intensity of the water diffusion in any direction at a certain point. The water diffusion in the brain is highly affected by its cellular organization. In particular, the axonal cell membranes and myelin sheaths are the main components restricting water mobility (Beaulieu, 2002).

00 .

\footnotetext{
* Corresponding author. Tel.: +4121693 36 32; fax: +412169376

E-mail address: lisa.jonasson@epfl.ch (L. Jonasson).

URL: http://lts5www.epfl.ch/.
}

Consequently, the measured DT becomes highly anisotropic and oriented in areas of compact nerve fiber organization, providing an indirect way of identifying fiber tracts. Analyzing such images can lead to a better understanding of white matter diseases, such as multiple sclerosis (Filippi et al., 2001; Maldjian and Grossman, 2001), schizophrenia (Lim et al., 1999; Foong et al., 2000) and dyslexia (Klingberg et al., 2000).

The DT is normally interpreted by calculating its eigenvalues and eigenvectors, the eigenvector corresponding to the highest eigenvalue describes the direction of the principal diffusion and the eigenvalue is a quantitative measure of the diffusion in that direction. Most of the existing methods for tracking fibers rely on the direction of principal diffusion to create integral curves describing the fiber paths (Conturo et al., 1999; 
Vemuri et al., 2001; Basser et al., 2000). Other methods use a probabilistic approach to explore more of the information contained in the diffusion tensor like, for example Hagmann et al. (2003), who consider the tensor as a probability distribution. Parker et al. (2001) have used level set theory, applied using fast marching methods, to find the connection paths between different brain regions. Campbell et al. (2002) have also used level set theory to implement a geometrical flow to track the fibers. They mostly focus on the problem of preventing leakage from the thin tubular structure that represents the fibers, by using flux maximizing flows.

Batchelor et al. (2001) use more of the tensor information by solving the diffusion equation iteratively. The method creates paths that originate from a chosen seed-point and can be considered as probability measures of a connection. A similar approach is presented by O'Donnell et al. (2002), where they find the steady state of the diffusion equation to create a flux vector field. In the same paper they show how the inverse diffusion tensor can define a Riemannian metric that is used to find geodesic paths that can be interpreted as fiber tracts.

The above methods focus on finding individual fiber paths whereas we have chosen to search for regions that correspond to certain fiber tracts, following the same idea as Tench et al. (2002). Tench segments regions by finding trajectories of individual fibers and then identifying voxels that belong to the same structure. The identification is based on the similarity of the shape of the trajectory paths. Zhukov et al. (2003) also focus on segmenting white matter regions from DT-MRI instead of on fiber tracts. They present new invariants of the tensor such as an anisotropy measure of the tensor that is rotationally invariant and not dependent on the computation of eigenvalues. These invariants are then used to calculate scalar volumes, one that represents the total diffusivity within a voxel and another that describes the anisotropy. On these scalar maps he segments regions with similar diffusion properties and then he applies a level set method to obtain a smooth segmentation. A drawback with Zhukov's method is that he only uses a scalar measure and ignores the directional information.

Very recent papers have addressed the problem of region based segmentation of DT-MRI using level set methods (Wang and Vemuri, 2004; Rousson et al., 2004; Feddern et al., 2003). The method presented by Wang and Vemuri (2004) uses the whole tensor information to define a region based force as proposed by Paragios and Deriche (1999). Although interesting, the method does not solve the problem of segmenting fiber tracts as a whole. Fiber tracts can not be seen as one region due to changes of the properties of the diffusion throughout the tracts.

Our method is using a 3D geometric flow designed to evolve inside the fiber tracts. By fiber tracts we mean re- gions containing compact and coherent bundles of axons. These can be tubular structures such as the cortico spinal tract as well as planar structures such as the corpus callosum. The white matter of the brain consists of all such different tracts. We segment these tracts by assuming that adjacent voxels in a tract have similar properties of diffusion. Therefore, we will measure the similarity of diffusion between voxels and then using this similarity as a front propagation speed. This means that the propagation speed of the front in a certain direction is proportional to the similarity between the tensors lying on the surface and their neighbors in that direction of propagation. The implementation of the geometric flow permits a local comparison between the voxels in the direction of the front propagation. When the fiber tracts turn, bend and change direction, this local comparison is important since the directional properties of the diffusion will not be similar in different locations along the fiber tract. The geometric flow does not only manage to evolve along the tracts but also towards the sides of it to fill it completely. Traditional methods of tractography often have a problem of filling whole tracts since these methods are very dependent on the initial points and only evolve along the fibers in the direction of diffusion. The measure of similarity that we use to compare the diffusion is based on the whole diffusion tensor, which helps to avoid problems that occur when only the principal direction is used. Due to noise in the acquisition, the order of the eigenvalues can sometimes be swapped but the measure of similarity that we use, the tensor scalar product (TSP), uses all possible combinations of the eigenvectors and an eventual swapping of principal directions will not influence the result. The influence of noise will thereby be diminished and it is also of importance for coping with the problem of fiber crossings where the principal direction loses its significance (Basser and Pajevic, 2000). As mentioned before, it is very important that we use the directional information as well as the quantitative information about the diffusion. In many regions of the brain, fiber tracts pass close to each other and if only a scalar value such as an anisotropy measure is used nothing will separate the regions from each other. Using the full tensor is necessary for satisfying results and it has already been shown for regularization problems (Chefd'hotel et al., 2002; Coulon et al., 2004; Tschumperle and Deriche, 2001; Vemuri et al., 2001).

The front propagation is implemented using the level set method as proposed by Osher and Sethian (1988). It assures a stable and accurate evolution. It handles natural topology changes and provides an elegant tool for smoothing the segmented tracts. While many methods demand a regularized tensor field, our geometric flow regularizes the 3D surface as it evolves inside the fiber tracts so that a regularized tensor field is not needed. This is made by a curvature dependent smoothing term 
which is called the minimal principal curvature. It is adapted for thin tubular structures as presented by Lorigo et al. (1999) for segmentation of blood vessels from MR angiography, and by not considering each tensor individually but also looking at its surroundings.

In neuroscience, various problems can benefit from such segmentation of the fiber tracts for applications such as getting a quantitative measure of the diffusion inside the chosen fiber tracts, white matter registration and surgical planning.

The paper is organized as follows. First we briefly present the concept of diffusion tensors and basic theories on front propagation with level set implementation. We then show how to use similarity measures for diffusion tensors to propagate a surface and how this can be used for fiber tract segmentation. The validation is made on synthetic images and we show segmentation results on real DT-MRI of the brains of two healthy subjects.

\section{Background theory}

\subsection{Diffusion tensor imaging and tensor similarity measures}

DT-MRI permits in vivo measures of the self-diffusion of water in living tissues. The tissue structure affects the Brownian motion of the water molecules which leads to an anisotropic diffusion that is measured by diffusion weighted MRI along at least six independent axes. A reference image without diffusion weighting is also required. As a second order approximation, the measured anisotropic motion can be modelled by an anisotropic Gaussian, that can be parameterized by the diffusion tensor in each voxel (Basser et al., 1994) to create a $3 \mathrm{D}$ field of diffusion tensors.

The DT is a $3 \times 3$ symmetric, semi-positive definite matrix. By diagonalizing the DT we obtain the eigenvalues $\left(\lambda_{1}, \lambda_{2}, \lambda_{3}\right.$, where $\left.\lambda_{1} \geqslant \lambda_{2} \geqslant \lambda_{3}\right)$ and the corresponding eigenvectors $\left(\hat{e}_{1}, \hat{e}_{2}, \hat{e}_{3}\right)$. Since the tensor is symmetric and semi-positive definite the eigenvalues are always non-negative. However, noise can destroy the semi-positivity of the measured DTs. The DT can be described in terms of its eigenvalues and eigenvectors.

$D=\left(\hat{e}_{1} \hat{e}_{2} \hat{e}_{3}\right)\left(\begin{array}{ccc}\lambda_{1} & 0 & 0 \\ 0 & \lambda_{2} & 0 \\ 0 & 0 & \lambda_{3}\end{array}\right)\left(\hat{e}_{1} \hat{e}_{2} \hat{e}_{3}\right)^{\mathrm{T}}$.

The largest eigenvalue and its corresponding eigenvector describe the quantity and direction of the principal diffusion.

Alexander et al. (1999) have explored many similarity measures for tensors to perform elastic matching of diffusion tensor images. These measures take both the magnitudes and the directions of the diffusion into ac- count. One of the most common measures of similarity between two tensors is the tensor scalar product (TSP). This is a measure of the overlap between two tensors:

$D_{1}: D_{2}=\operatorname{Trace}\left(D_{1} D_{2}\right)=\sum_{j=1}^{3} \sum_{i=1}^{3} \lambda_{1 i} \lambda_{2 j}\left(e_{1 i} e_{2 j}\right)^{2}$.

The TSP is often normalized to avoid influence by the relative size of the two tensors. This will emphasize the shape and orientation of the tensor

$\operatorname{NTSP}\left(D_{1}, D_{2}\right)=\frac{D_{1}: D_{2}}{\operatorname{Trace}\left(D_{1}\right) \operatorname{Trace}\left(D_{2}\right)}$.

Another way of measuring the tensor similarity using the full tensor information that is also presented by Alexander et al. (1999), is the tensor difference (TD).

$\operatorname{TD}\left(D_{1}, D_{2}\right)=\sqrt{\left(D_{1}-D_{2}\right):\left(D_{1}-D_{2}\right)}$,

where $\ldots . \ldots$ is given as in (2). The TD measures the difference in size and shape as well as orientation between the two tensors.

The diffusion in the direction of a unit vector, $\hat{x}$, is given by the double contraction of the DT with the vector:

$d(\hat{x})=\hat{x} D \hat{x}$.

A way of directly comparing the diffusion between two tensors is to compare the diffusion in the direction of all unit vectors on a sphere, $\hat{x}$, using the double contraction. We will call this measure of similarity for integral similarity (IS).

$\operatorname{IS}\left(D_{1}, D_{2}\right)=\int \min \left(\frac{d_{1}(\hat{x})}{d_{2}(\hat{x})}, \frac{d_{2}(\hat{x})}{d_{1}(\hat{x})}\right) d \hat{x}$,

where $d_{1}(\hat{x})$ is the diffusion in direction $\hat{x}$ for the diffusion tensor $D_{1}$. The IS gives us a percentage of the common diffusion for the two tensors.

\subsection{Geometric flows and level set implementation}

Geometric flows and especially curvature- or curve shortening flows are becoming more and more important regularization tools in computer vision. A curvature flow is a curve or surface that evolves at each point along its normal with a velocity depending on the curvature at that point. This process leads to a smoothing of the curves or surfaces to eliminate the effects of noise. The theory is well developed for the two dimensional case and even though some of the properties of the 2D curve, such as the property of shrinking to a point under mean curvature flow, do not hold in the $3 \mathrm{D}$ case, the main part of the theories remains valid and works well for segmentation of $3 \mathrm{D}$ objects.

To use the geometric flows for image segmentation, the evolution of the curve or surface has to depend on external properties determined by the image features. A classical speed function to segment gray scale images 
is based on the gradient of the images and goes to zero when the surface approaches an edge (Caselles et al., 1997).

A general flow for a 3D closed surface can be described as

$\frac{\partial S}{\partial t}=(F+\mathbf{H}) N$,

where $F$ is an image based speed function, $\mathbf{H}$ is an intrinsic speed dependent on the curvature of the surface, $S$ is the surface, $N$ is the normal of the surface and $t$ is the time.

To solve this time dependent partial differential equation (PDE) we use the level set method, introduced by Osher and Sethian (1988), where the evolving surface is considered as a constant level set of a function of a higher dimension. By doing this we obtain a numerically stable algorithm that easily handles topology changes of the evolving surface. In our case the function of higher dimension is the signed distance function, $\phi(t)$, of the evolving surface. Finally, the evolution of the zero level set of $\phi$, which coincides with the surface $S(t)$, is described by

$\frac{\partial \phi}{\partial t}=(F+\mathbf{H})|\nabla \phi|$.

\section{Method}

\subsection{Similarity based front propagation}

As mentioned in the introduction we propose a front propagation method that is based on the assumption that the diffusion is similar between two adjacent voxels within the same tract. To perform the segmentation a small initial surface is placed inside the tract we wish to segment and the surface is then propagated using the similarity measure in (3). The front propagates into a voxel with a speed proportional to the similarity between the diffusion tensor in the voxel and the diffusion tensors in the adjacent voxels lying inside the fiber. We define the front propagation speed as

$F=\operatorname{mean}\left(\operatorname{NTSP}\left(D_{i}, D_{i-1}\right), \operatorname{NTSP}\left(D_{i}, D_{i-2}\right)\right)$,

where NTSP is the normalized tensor scalar product as in (3). $D_{i}$ is the DT in the current voxel and $D_{i-p}$ are the DTs in voxels found by following the normal to the surface $p$ voxels backwards from the original voxel $i$, see Fig. 1, and then select the nearest neighbor.

Different measures of similarity such as the TD (4) and the IS (6) have also been analyzed. Finally we have found the NTSP most suitable for our purposes. Firstly, it is dependent on the global shape of the tensor since it takes into account all eigenvalues and eigenvectors which is not the case with the other similarity measures.

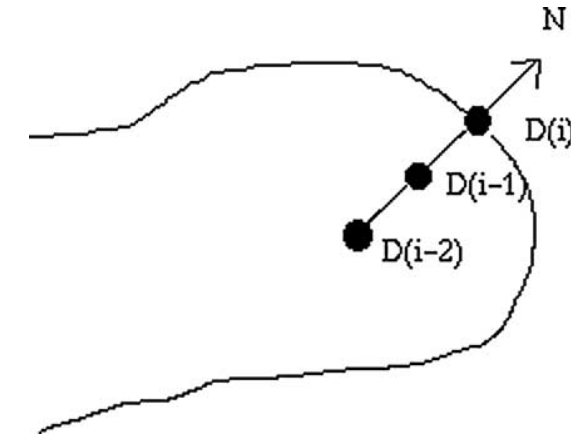

Fig. 1. Choice of adjacent voxels with respect to the normal of the surface.

Secondly, since the main objective is to propagate the surface in the anisotropic regions while avoiding the isotropic regions, we want a high speed, $F$, in the anisotropic areas and a low speed in the isotropic areas. For this, the NTSP measure is highly interesting since only a completely anisotropic tensor with diffusion in only one direction compared with itself will sum up to one and the similarity between two isotropic tensors are low (see Table 1). Thirdly, this similarity measure is robust with respect to noise. Noise can sometimes swap the order of the eigenvalues but since the NTSP combines eigenvalues and eigenvectors independently of the order, this measure is more stable compared to other measures.

It is important to notice that the presented flow does not necessarily evolve in the direction of the diffusion. It evolves in the direction where the diffusion properties do not differ too much from the local neighborhood inside the fiber. This allows the surface to propagate towards the sides of the fiber tract and thereby segment the whole tract.

\subsection{Regularization}

Due to a high level of noise in the DT-MRI a segmentation only based on properties of the diffusion will be very irregular. To smooth the tracts while segmenting them we regularize the flow by adding a curvature dependent speed. Lorigo et al. (1999) introduced the

Table 1

Comparison between similarity measures between different combinations of isotropic (17) and anisotropic (18) tensors

\begin{tabular}{llll}
\hline Tensors & NTSP & 1-TD & IS \\
\hline$\left(D_{\text {anisotropic,0 }}, D_{\text {anisotropic, } 0}\right)$ & 0.57 & 1 & 1 \\
$\left(D_{\text {isotropic }}, D_{\text {isotropic }}\right)$ & 0.33 & 1 & 1 \\
$\left(D_{\text {isotropic }}, D_{\text {anisotropic, } 0}\right)$ & 0.33 & 0.25 & 0.54 \\
$\left(D_{\text {anisotropic, },}, D_{\text {anisotropic,30 }}\right)$ & 0.51 & 0.75 & 0.79 \\
$\left(D_{\text {anisotropic,0},}, D_{\text {anisotropic,45 }}\right)$ & 0.46 & 0.5 & 0.72 \\
$\left(D_{\text {anisotropic, },}, D_{\text {anisotropic,90 }}\right)$ & 0.36 & 0 & 0.64 \\
\hline
\end{tabular}

The normalized tensor scalar product (NTSP), the tensor difference (TD) and integral similarity (IS). Here $D_{\text {anisotropic, } n}$ represents the anisotropic tensor with its principal vector rotated $n$ degrees. 
use of a curvature definition from co-dimension 2 flows on surfaces with a thin, tubular structure. Instead of using either mean curvature or gaussian curvature, which normally destroy the tubular structure, they use the minimal principal curvature which is a combination of both curvatures. The minimal principal curvature, $\kappa$, is given by

$\kappa=H-\sqrt{H^{2}-K}$,

where $H$ is the mean curvature and $K$ is the gaussian curvature. For the definitions of the mean- and gaussian curvature we refer to (Sethian, 1999). This definition of the curvature smoothes the tubes axially and not radially, as if they were open curves in a 3D space, instead of smoothing their tubular form. Thus, this curvature flow satisfies the property of shrinking to a point while preventing undesirable changes of topology. We use this smoothing model for our curvature dependent smoothing term. The minimum principal curvature flow does not only smooth tubular structures but also surfaces in general while allowing smaller structures to stick out. This makes it possible for a thin fiber tract to evolve independently away from a larger structure.

Our geometric flow now has the form

$\frac{\partial S}{\partial t}=(F+\kappa) N$

This can easily be implemented with the level set method.

\section{Parameters and implementation details}

The method has been implemented in Matlab 6.1 (The MathWorks, Inc.) except for the reinitialization of the signed distance function, which has been implemented in $\mathrm{C}$ and compiled with the mex-library, so the function can be called from Matlab.

\subsection{Preserving the signed distance function (SDF)}

As mentioned in Section 2.2 the evolving surface is considered as the zero level set of its SDF, $\Phi$. Due to local dependence of the propagation speed, the evolution of the other level sets differs from the zero level set. This creates irregularities that deform $\Phi$ so it ceases to be a signed distance function. A correct SDF is crucial to get a correct and smooth evolution of the surface, since the calculations of the normals and curvatures depend directly on the SDF. Therefore, a reinitialization of the signed distance map is made at every iteration. It is implemented using the fast marching method to solve the time dependent PDE (Adalsteinsson and Sethian, 1995): $\frac{\partial \phi}{\partial t}=\operatorname{sign}\left(\phi_{0}\right)(1-|\nabla \phi|)$.

During the evolution process it is very important to preserve the SDF to assure a correct computation of the normals and the minimal principal curvature. If we do not reinitialize the level set function the propagation quickly becomes unstable.

Furthermore, we carry out an extension of speed values from the zero level set to a narrow band to reduce the perturbations of the SDF. Indeed, closest to the zero level set we easily get discontinuities since the speed function can vary a lot between two adjacent voxels. It is also in this area that a correct SDF is of most importance, since the exact position of the zero level set is dependent on the values of the surrounding voxels. To assure the maintenance of the SDF, the speed is calculated only on the zero level set and is then transferred in the direction of the normal to the voxels lying just next to the zero level set.

\subsection{Thresholding}

If the speed at one voxel is not equal to zero it will eventually lead to a propagation of the front at that voxel, even though the speed might be very small. To prevent unwanted propagation all speeds inferior to a certain threshold are set to zero. Thresholding is a very abrupt method so it risks causing discontinuities in the propagation. Since it is very important to maintain a continuous speed, we use the regularized Heaviside function, defined in (Sussman et al., 1998), to get a smoother thresholding.

$$
H_{T}(x)= \begin{cases}0 & \text { if } x<T-\epsilon, \\ \frac{1}{2}\left[1+\frac{x-T}{\epsilon}+\frac{1}{\pi} \sin (\pi(x-T) / \epsilon)\right] & \text { if }|x-T| \leqslant \epsilon \\ 1 & \text { if } x>T+\epsilon\end{cases}
$$

where $T$ is the selected threshold and $\epsilon$ is equal to 0.1 in all experiments.

The surface evolution is stopped when the propagation speed has been sufficiently small for several successive iterations.

\subsection{Weighting the speed terms}

The diffusion dependent and the curvature dependent speed is not always of the same order. To have a satisfactory regularization without inhibiting the front propagation it is therefore important to set the weighting factor between them correctly. The curvature term in (11) is then referred to as $\alpha \kappa N$,

$\frac{\partial S}{\partial t}=(F+\alpha \kappa) N$ 
where $\alpha$ is the weighting parameter. In all our experiments $\alpha$ is set to 0.1 .

Finally, our surface evolution equation has the following form:

$\frac{\partial S}{\partial t}=\left(H_{T}(F)+\alpha \kappa\right) N$,

and the evolution of its level set function is

$\frac{\partial \Phi}{\partial t}=\left(H_{T}(F)+\alpha \kappa\right)|\nabla \Phi|$.

In the annex we prove the existence and uniqueness of a solution for this PDE.

\section{Validation and results}

\subsection{Synthetic tensor fields}

Synthetic tensor fields have been created to test the method. Tensor values for one isotropic and one anisotropic tensor were taken from real data on DT-MRI of the brain of a healthy subject. The values of the anisotropic tensor have then been manipulated so that the principal diffusion is in the $x$-direction. The values of the isotropic tensor and anisotropic tensor are the following:

$D_{\text {isotropic }}=\operatorname{diag}(3,3,3) \cdot 10^{-4}$,

$D_{\text {anisotropic }}=\operatorname{diag}(7,2.5,0.4) \cdot 10^{-4}$.

The values are given in $\mathrm{mm}^{2} / \mathrm{s}$.

Uniformly distributed random noise is added to the isotropic tensors so all of them will not be identical, these are used as a background for the fibers that are modelled using the anisotropic tensors. To obtain the desired direction of the anisotropic tensors they are rotated by first multiplying the principal diffusion vector by a rotation matrix and then projecting the remaining eigenvectors onto a plane which is orthogonal to the new principal direction of diffusion. The original eigenvalues are kept and the tensors are then reconstructed according to (1).

With this method two different 3D tensor fields are constructed, presented in Fig. 2. The images show the largest eigenvector of the tensors at a cut along the $z$ axis. The first tensor field shows a semicircle to demonstrate the ability of following a curved fiber tract. The second tensor field simulates a branching fiber tract.
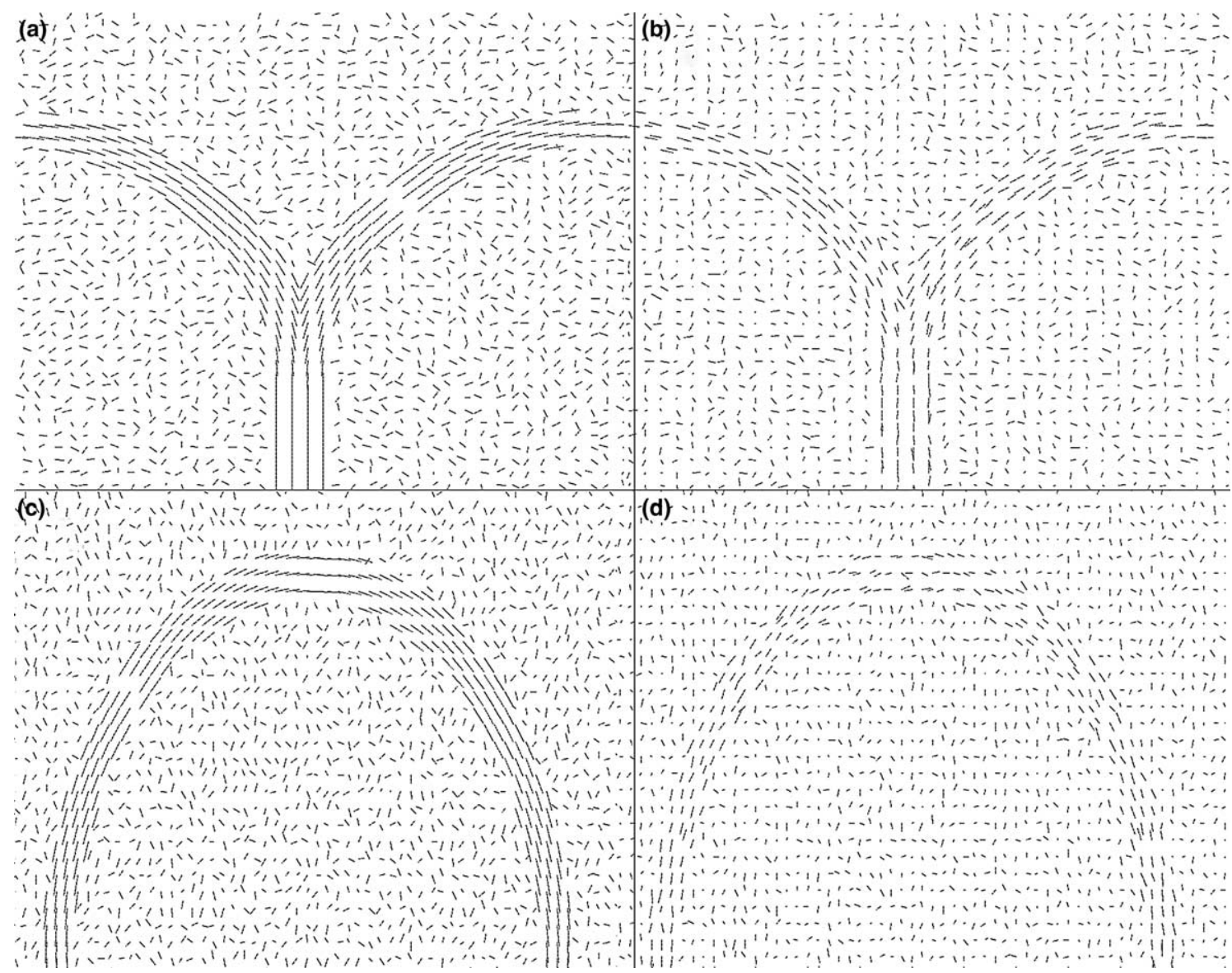

(d)

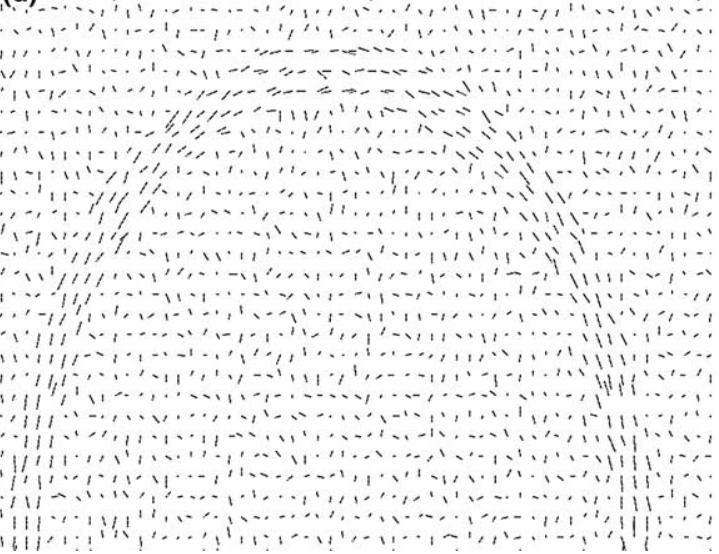

Fig. 2. Synthetic DT fields modelling a diverging and a curved fiber tract. The principal directions of diffusion on a cut along the $z$-axis. (a, c) Before noise is added. $(b, d)$ After noise is added. 
To make the tensor fields more realistic, noise is added (Tournier et al., 2002). The six amplitude images from which the diffusion tensors originally would have been acquired in DT-MRI are obtained by making the inverse calculation and then noise is added on the amplitude images. The added noise is an approximation of $\mathrm{Ri}-$ cian noise (Gudbjartsson and Patz, 1995; Henkelman, 1985 ) as it would be on MR data. Then the tensor images are recreated. The resulting tensor fields can be seen in Fig. 2.

The method is tested on the synthetic images with different levels of SNR. We have used a SNR of 8, 16 and 32 on the MR amplitude images as in (Tournier et al., 2002). To start the segmentation a small initial surface is placed somewhere inside the synthetic fiber. To give an idea of suitable thresholds we here present some typical values of the NTSP between different combinations of the tensors presented above. In the same table we also present corresponding values for different similarity measures.
Correct segmentations were obtained with several different thresholds between 0.45 and 0.5 . These thresholds are used on the real MR data. In Fig. 3, the effects of different choices of thresholds are shown. All synthetic tensor fields used for the segmentation have a $\mathrm{SNR}=8$. Even though the synthetic tensor fields are very noisy, the resulting surfaces are relatively smooth due to our regularization that is performed as the surface is evolving. In Fig. 4, the level of regularization is varied. It shows the segmentation results without regularization term, with a regularization term set too high and with an appropriate value of the regularization term.

In Tables 2 and 3, quantitative values for the segmentation results are presented. The value is obtained by comparing the signed distance function between the contour of the synthetic fiber tract without noise and the segmented fiber tract when noise is added with $\mathrm{SNR}=8$, and then calculating the error between the SDFs at the contours. The tables present the mean $\left(L_{1}\right)$ and maximum $\left(L_{\infty}\right)$ differences in value between the

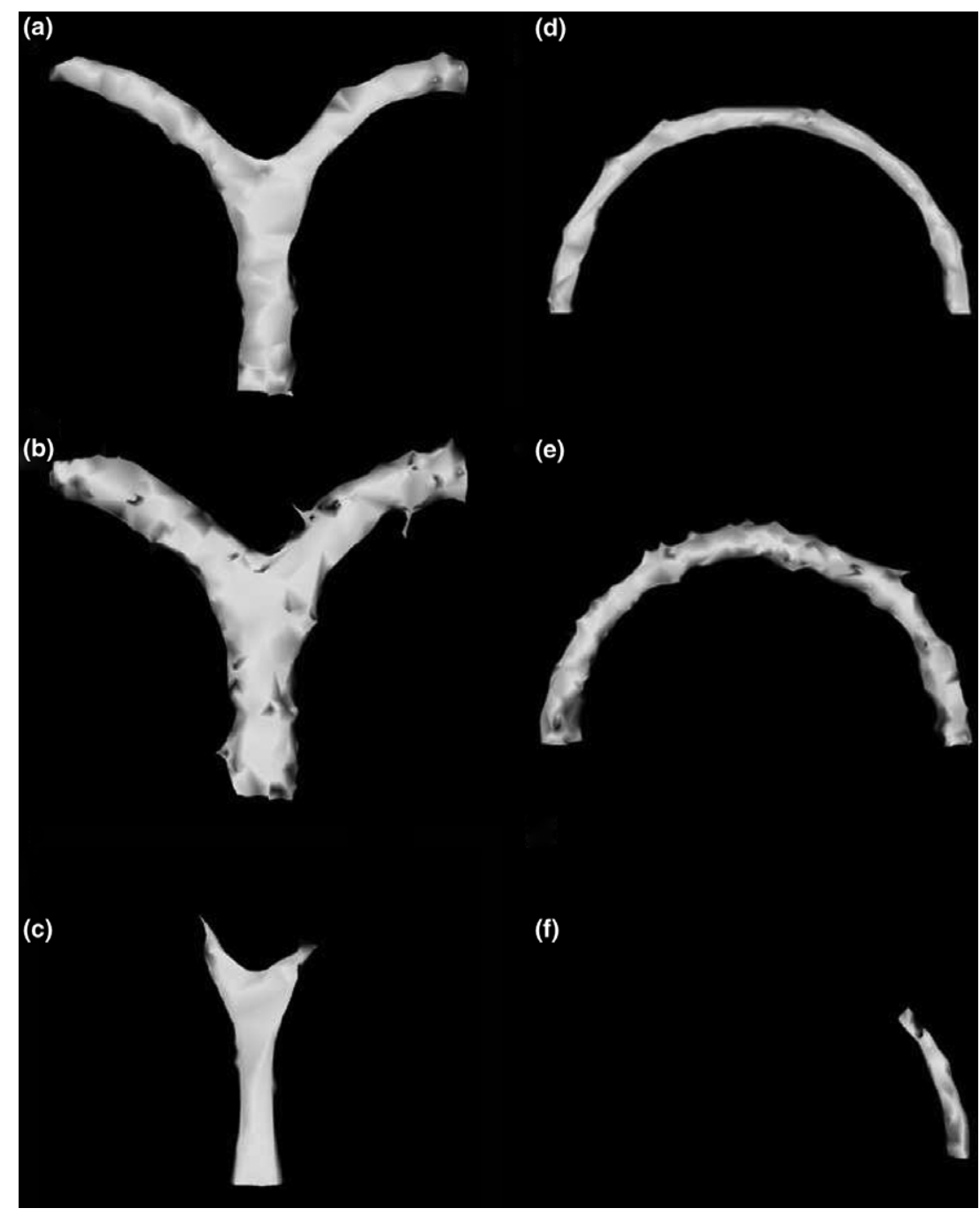

Fig. 3. Effects of varying thresholds on the resulting segmentations of synthetic DT fields with SNR $=8$. (a, d) Segmentation is ideal with threshold $=0.45$. (b, e) Segmentation is too large with threshold $=0.40,(\mathrm{c}, \mathrm{f})$ Segmentation is insufficient with threshold $=0.55$. 


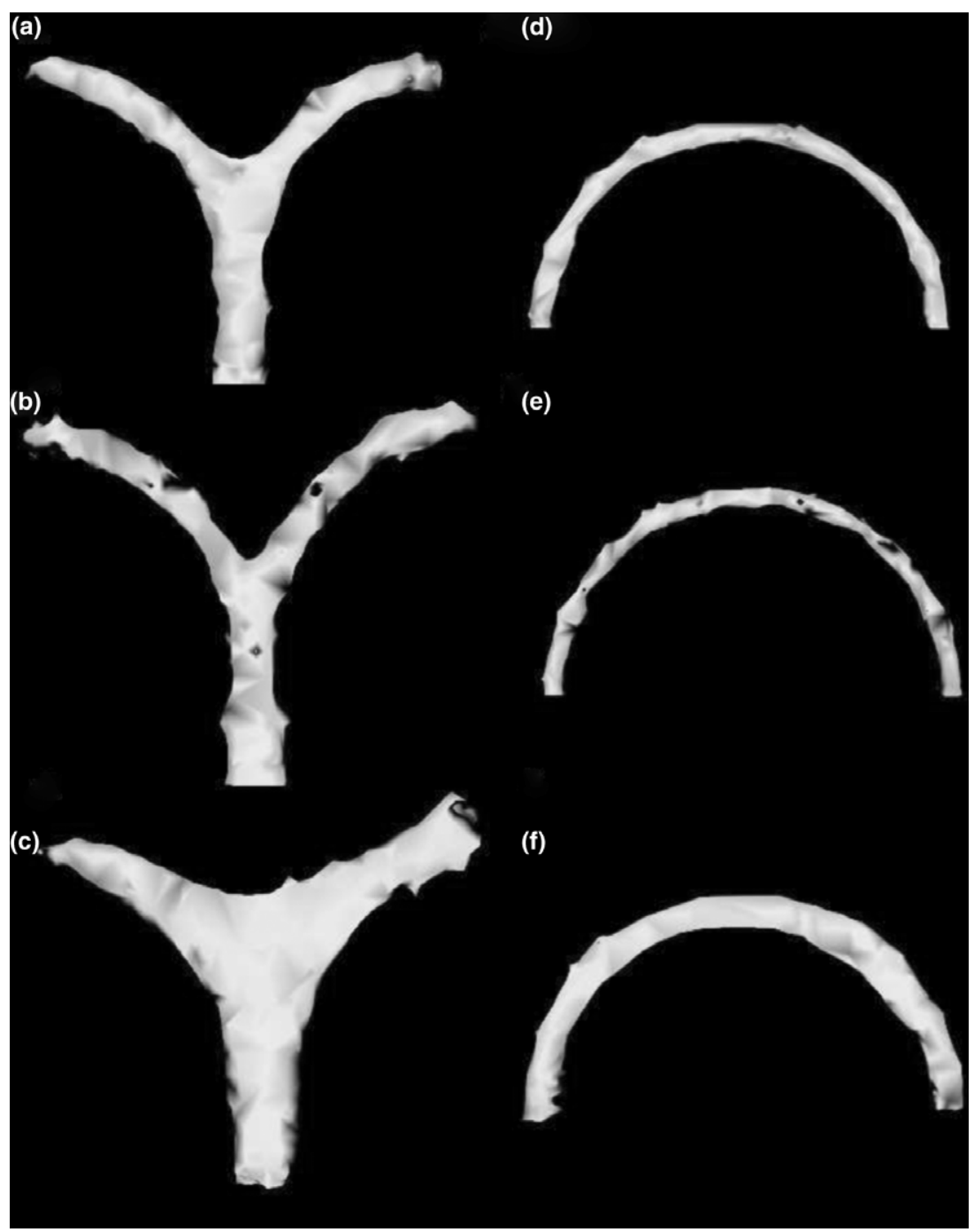

Fig. 4. Effects of varying regularization parameter on the resulting segmentations of synthetic DT fields with SNR $=8$. (a, d) Ideal regularization parameter. (b, e) No regularization, the segmentation is too noisy. (c, f) Regularization parameter is set too high and has too high an influence on the real shape of the tracts. Threshold $=0.45$ for all images.

two SDFs. Table 2 shows the results for different thresholds and Table 3 for different weighting of the regularization parameter. Both tables confirm the visual results that the best results are obtained for a threshold equal to 0.45 and a weighting factor for the curvature $\alpha=0.1$.

Table 2

Quantitative values of the segmentation results for different thresholds

\begin{tabular}{lllcc}
\hline Form & Threshold & $\alpha$ & Max & Mean \\
\hline Circle & 0.40 & 0.1 & 2.98 & 0.56 \\
& 0.45 & 0.1 & 2.1 & 0.48 \\
& 0.55 & 0.1 & 47.5 & 20.5 \\
Fork & 0.40 & 0.1 & 3.96 & 0.63 \\
& 0.45 & 0.1 & 1.56 & 0.51 \\
& 0.55 & 0.1 & 17.8 & 6.0 \\
\hline
\end{tabular}

The values are obtained by comparing the signed distance functions of the contour of the synthetic fiber tract without noise and those of the segmented fiber tract when noise is added with a $\mathrm{SNR}=8$.
Experiments have also been made with smaller and larger neighborhood than in (9). For a smaller neighborhood the front propagation becomes too sensitive to noise. A larger neighborhood, in the sense that we look

Table 3

Quantitative values of the segmentation results using different levels of regularization

\begin{tabular}{lllll}
\hline Shape & Threshold & $\alpha$ & Max & Mean \\
\hline Circle & 0.45 & 0 & 2.32 & 1.07 \\
& 0.45 & 0.1 & 2.1 & 0.48 \\
& 0.45 & 2 & 3.3 & 0.29 \\
Fork & 0.45 & 0 & 2.0 & 1.06 \\
& 0.45 & 0.1 & 1.56 & 0.51 \\
& 0.45 & 2 & 2.69 & 0.6 \\
\hline
\end{tabular}

The values are obtained by comparing the signed distance functions of the contour of the synthetic fiber tract without noise and those of the segmented fiber tract when noise is added with a $\mathrm{SNR}=8$. 
further behind the surface in the opposite direction of the normal, improves slightly the robustness of the method but the flow has difficulties segmenting bending fiber tracts and it also increases the computation time. A larger neighborhood, in the sense of adding voxels on the side of the normal, would make it impossible to track thinner fiber tracts. The best compromise between robustness and correctness of the segmentation is a neighborhood, lying on a line in the opposite direction of the normal, of two voxels.

\subsection{Real DT-MRI}

\subsubsection{MRI data acquisition}

The diffusion tensor images used here were acquired with a $1.5 \mathrm{~T}$ clinical MRI scanner (Magnetom Symphony; Siemens, Erlangen, Germany). The data was produced with a diffusion-weighted single-shot EPI sequence using the standard Siemens Diffusion Tensor Imaging Package for Symphony. We acquired 44 axial slices in a $128 \times 128$ matrix covering the whole brain of healthy volunteers, from the vertex to the end of the cerebellum. The voxel size was $1.64 \mathrm{~mm} \times 1.64 \mathrm{~mm}$ with a slice thickness of $3.00 \mathrm{~mm}$ without gap. Timing parameters were a TR of $1000 \mathrm{~ms}$ and a TE of 83 ms. Diffusion weighting was performed along 6 independent axes and we used a b-value of $1000 \mathrm{~s} / \mathrm{mm}^{2}$ at a maximum gradient field of $30 \mathrm{mT} / \mathrm{m}$. A normalizing image without diffusion weighting was also acquired. In order to increase the signal-to-noise ratio the measures were repeated 20 times. An anatomical T1 3D gradient echo volume of the entire head was also acquired during the same session. The whole examination lasted about one hour. In the following example, images from two healthy volunteers (male and female between 25 and 30 years old) were considered. Informed consent was obtained in accordance with institutional guidelines.

\subsubsection{Preprocessing of data}

The preprocessing of the data and the geometric flow evolution was carried out in Matlab 6.1. The diffusion tensor was computed for each voxel by linear combination of the log-ratio images according to Basser and Pierpaoli (1998). The tensors were linearly interpolated component-wise between slices along the $z$-axis, to obtain a volume with a 3D regular grid of $1.64 \mathrm{~mm}$.

To place the initial surface for the front propagation, we use color images representing directional information according to a method presented by Pajevic and Pierpaoli (1999). The initial surface is then placed inside an anisotropic region belonging to the fiber tract we wish to segment. The initial surface can be as small as one voxel but in general we chose the initial surface to be a one voxel thin tube that is directed along the supposed fiber.

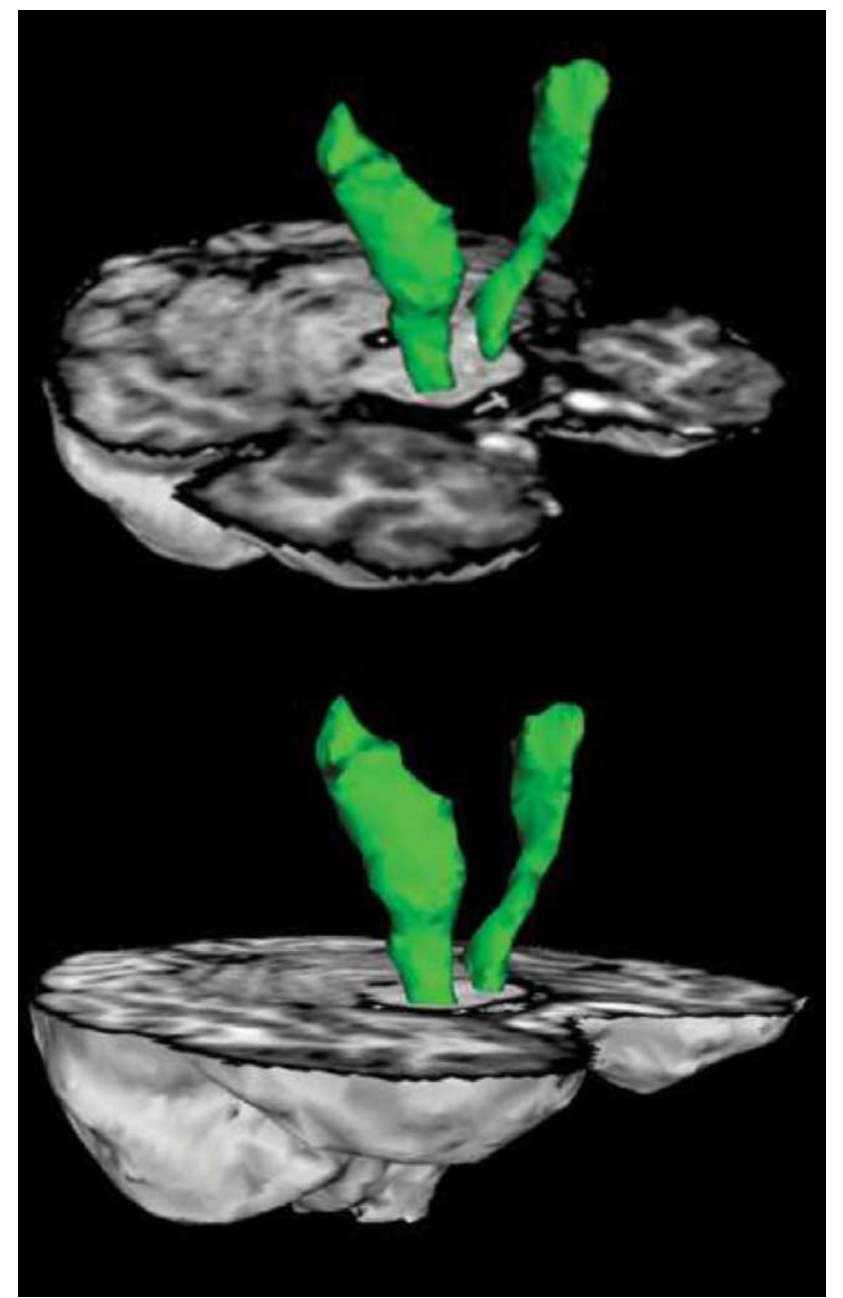

Fig. 5. Segmentation of the left- and right cortico-spinal tract.

\subsubsection{Results}

The segmentation has been performed on three different DT-MR images. Two of the image acquisitions are from the same person. The results have been validated visually by comparing with post-mortem based neuroanatomical knowledge. Results are presented for one of the DT-MR images.

On the synthetic images we saw that several different thresholds are possible for a good segmentation. On the real MR data the same range of thresholds has been used and depending on the segmentation we desire, the threshold has been slightly varied within this range. In Fig. 5, the cortico-spinal tract has been successfully segmented. To illustrate the effect of different choices of threshold on real DT-MRI, two segmentations of the corpus callosum have been made with different thresholds. The results can be seen in Fig. 6. For a stricter threshold only the medial part, i.e. the splenium, the truncus and the genu of the corpus callosum is segmented. When choosing a lower threshold the segmentation surface extends further towards the forceps major, 


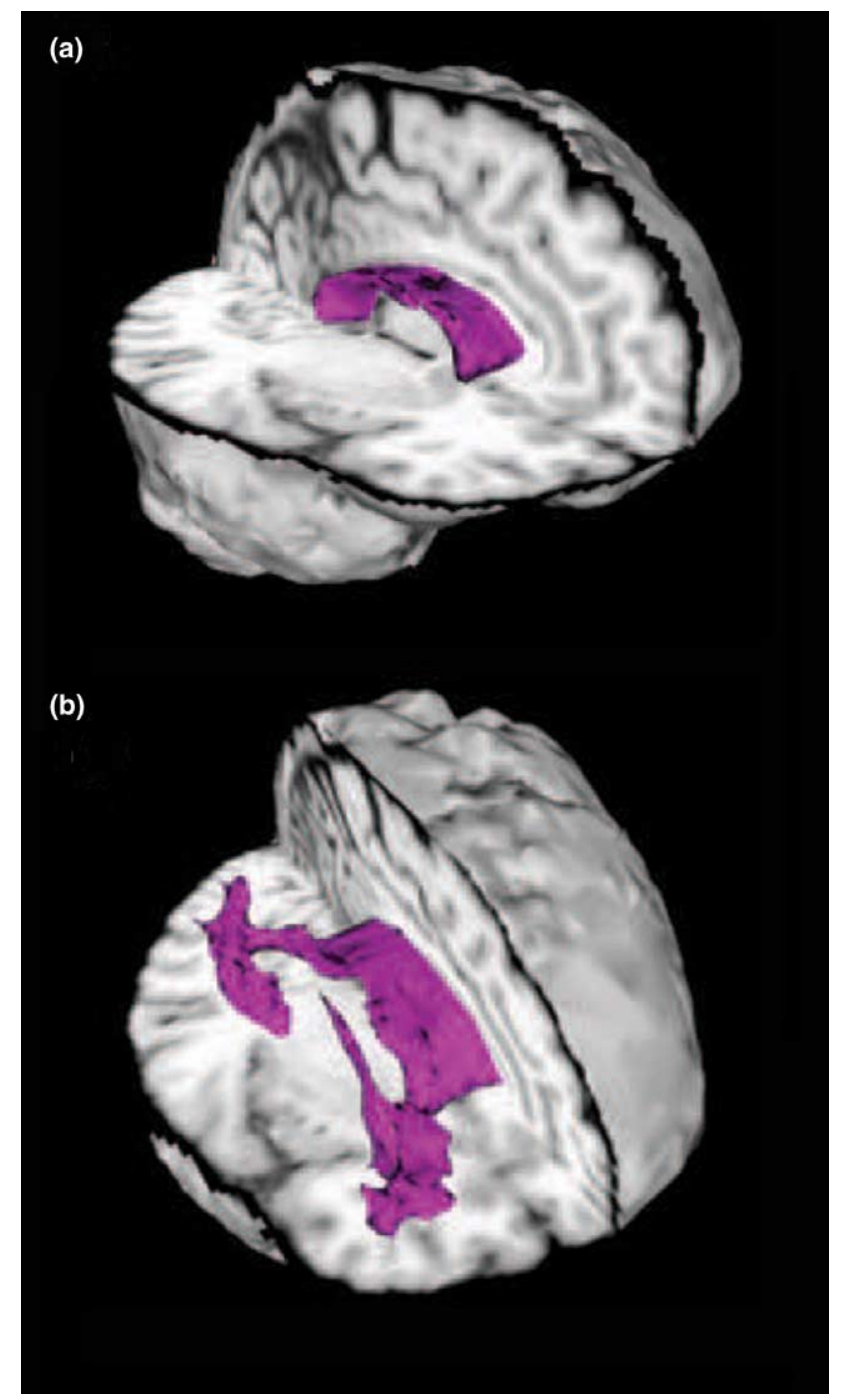

Fig. 6. Segmentation of the corpus callosum. (a) Segmentation is limited to the splenium, truncus and genu when threshold $=0.47$. (b) In addition to (a) the segmentation also includes the forceps major, the radiations, the tapetum and the forceps minor when threshold $=0.45$.

the radiation of the corpus callosum, the tapetum and the forceps minor.

In Fig. 7, we segmented the inferior long association bundles as a whole, containing the occipito-frontal, the longitudinal inferior and the uncinate fascicles. These bundles run in an intricate and parallel fashion for most of their trajectories, therefore we treat them as one entity. It is a good example of a case where the diffusion orientation plays a crucial role since no contrast between this group and adjacent structures exists neither on fractional anisotropy maps, T1 or T2 images.

\section{Discussion and conclusion}

We have presented a new method for segmentation of the main core of fiber tracts by assuming that two adja-

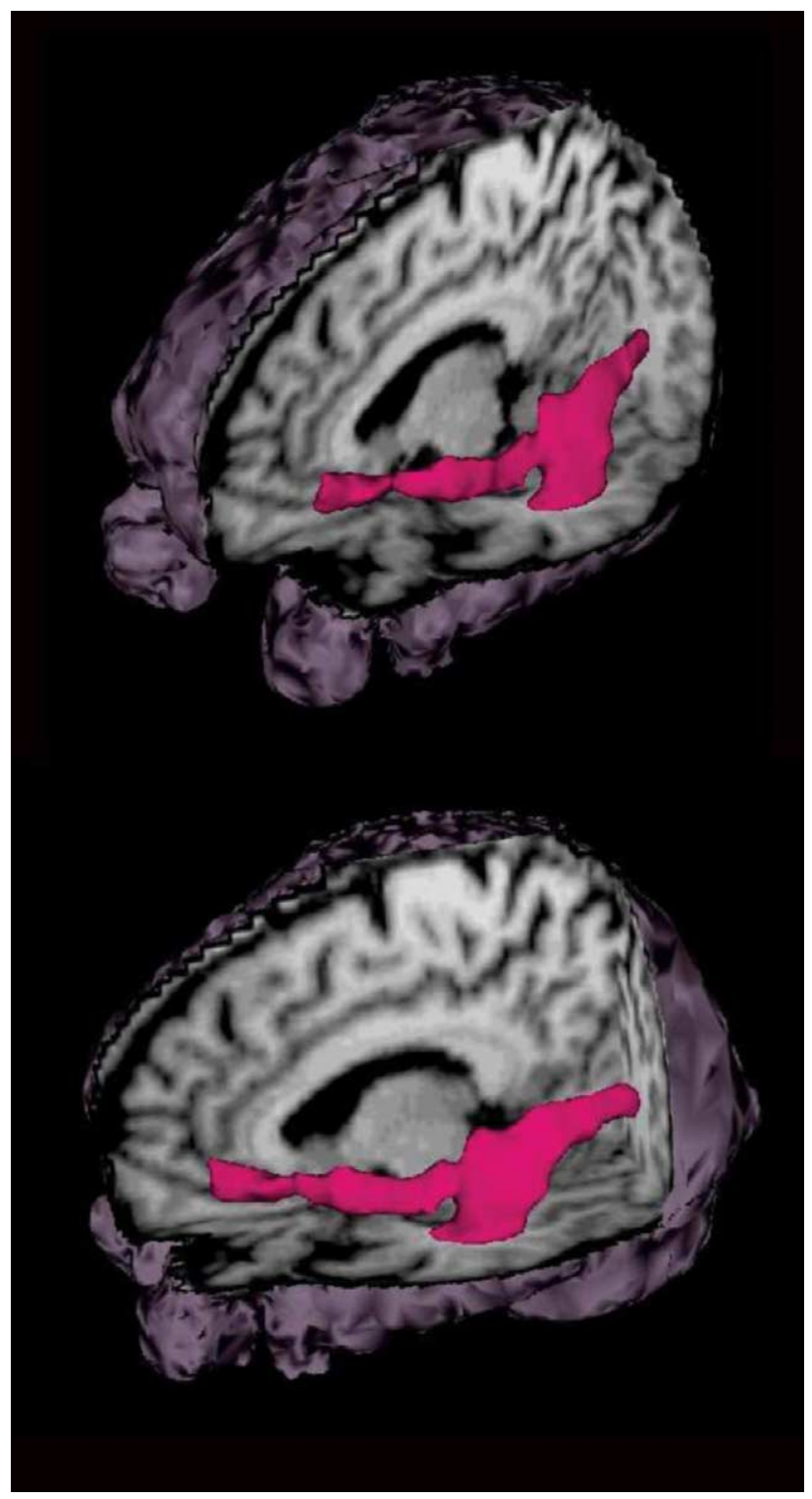

Fig. 7. Segmentation of the inferior long association bundles as a whole (occipito-frontal, longitudinal inferior and uncinate fascicles).

cent voxels within the same tract have similar diffusion properties. The method manages to segment the larger tracts in the brain. This presented segmentation methodology can be very useful for several purposes such as studying the water diffusion in a tract of interest, e.g. the genu of the corpus callosum and the centrum semiovale in alcoholics (Lim and Helpern, 2002; Pfefferbaum and Sullivan, 2002) or in the splenium of the corpus callosum in schizophrenic patients (Foong et al., 2000). Through its precise description of the contour of a white matter structure it allows us to compare its shape across a selected population, e.g. study of the corpus callosum sexual dimorphism (Clarke et al., 1989; Pettey and Gee, 2002). 
The segmentation results are sensitive of the choice of parameters. Since there is no objective measure of the exact solution on the brain images it is difficult to determine exactly the optimum threshold. This is not necessarily a negative property. An advantage is the flexible segmentation, when the choice of threshold determines how far to go in the fibers as shown in Fig. 6.

The balance between the propagation force and the regularization force has also been determined on the synthetic images. We have seen that ignoring regularization leads to a noisy and irregular surface. When the regularization force is too strong the global shape of the segmented tract is deformed.

As mentioned in Section 1, most of the existing methods focus on following the principal eigenvector of the diffusion tensor. The diffusion tensor contains a lot more information than just the main direction and magnitude of the diffusion. The other eigenvectors and eigenvalues also contain important data which is often ignored. Just looking at the principal direction also leads to a larger sensitivity to noise since a smaller deviation of the principal direction will lead to an important accumulative error. By exploring more of the tensor information we have created a flow that is less sensitive to noise. The similarity measure we use is based on the whole tensor and combines all eigenvectors which makes it insensitive to swapping of eigenvalues, a phenomenon that can appear in the presence of noise. The similarity measure is shape dependent and favors anisotropic diffusion which is a great advantage for our application.

Using geometric flows for the segmentation has the advantage to other methods that it permits local comparisons of the diffusion in the direction of the surface evolution. An important advantage of our approach is the level set implementation. It provides an elegant tool for propagating and smoothing the segmented tracts and makes it possible to follow several paths simultaneously and effectively handle branchings and merging of fiber tracts.

Another advantage of the flow is that we normalize with the total diffusion in each point. This eliminates the influence of the diffusion strength and makes a more correct measure of the common diffusion of the tensors. Calculating the NTSP with adjacent voxels lying inside the propagating surface leads to a regularization of the fiber tract in addition to the regularization performed with the curvature based propagation force.

\section{Acknowledgements}

This work is supported by the Swiss National Science Foundation Grant Nos. 2153-066943.01 and 3235B0102868.

\section{Appendix A. Existence and uniqueness of our segmentation model}

This section deals with the mathematical study of our fiber tract segmentation model. We show that a unique continuous viscosity solution exists for our second order PDE:

$$
\left\{\begin{array}{l}
\phi_{t}+G\left(t, x, \nabla \phi, \nabla^{2} \phi\right)=0 \quad \text { in }(0, T] \times \mathbb{R}^{3}, \\
\phi(0, x)=\phi_{0}(x) \text { in } \mathbb{R}^{3},
\end{array}\right.
$$

where $\nabla$ stands for the gradient, $\nabla^{2}$ is the Hessian matrix and the function $G$ of our problem is

$G=-\left(H_{\mathrm{T}}(F)+\alpha \kappa\right)|\nabla \phi|$,

where $F$ is our front propagation speed defined in (9), based on the tensor diffusion, $H_{T}$, introduced in (13), is a $C^{2}$ function that approximates the Heaviside function, $\kappa$ is the minimal principal curvature of level sets of $\phi$ and $\alpha$ is a positive constant.

Our result is based on the theory of viscosity solutions (Crandall et al., 1992) and more precisely on the papers of Ambrosio and Soner (1996) for the minimal principal curvature flow and Chen et al. (1991) for generalized curvature flow equations. Viscosity solutions are used for the study of non-smooth solutions of PDEs. They are consistent with classical smooth solutions since they coincide with them when classical solutions exist.

Definition 1. [Monotonicity condition (Crandall et al., 1992)]. Let $u: \mathbb{R}^{n} \rightarrow \mathbb{R}$ a continuous real-valued function and $P\left(x, u, \nabla u, \nabla^{2} u\right)$ a partial differential equation such that $P: \mathbb{R}^{n} \times \mathbb{R} \times \mathbb{R}^{n} \times S^{n \times n} \rightarrow \mathbb{R}$, where $S^{n \times n}$ be the set of all symmetric, $n \times n$ matrices. The function $P$ is said to be proper if it satisfies

1. $P\left(x, u_{r}, p, X\right) \leqslant P\left(x, u_{s}, p, X\right)$ with $u_{r} \leqslant u_{s}$,

2. $P(x, u, p, X) \leqslant P(x, u, p, Y)$ with $X \geqslant Y$, where $u_{r}, u_{s} \in$ $\mathbb{R}, x, p \in \mathbb{R}^{n}, X, Y \in S^{n \times n}$. The second condition is called degenerate ellipticity.

Definition 2. [Viscosity solutions (Ambrosio and Soner, 1996)]. Let $u:[0, T) \times \mathbb{R}^{n} \rightarrow \mathbb{R}$ be a locally bounded function and $P:[0, T) \times \mathbb{R}^{n} \times \mathbb{R} \times \mathbb{R}^{n} \times S^{n \times n} \rightarrow \mathbb{R}$ be proper. For any function $v$, the upper semicontinuous (u.s.c.) envelope $v^{*}$ of $v$ is the smallest u.s.c. function that is greater than or equal to $v$. Similarly, the lower semicontinuous (1.s.c.) envelope $v_{*}$ of $v$ is the largest 1.s.c. function that is less than or equal to $v$.

(A) We say that $u$ is a viscosity subsolution of $u_{t}+P\left(t, x, u, \nabla u, \nabla^{2} u\right)=0$ in $(0, T) \times \mathbb{R}^{n}$ if for any $\varphi \in C^{2}\left((0, T) \times \mathbb{R}^{n}\right)$

$\varphi_{t}\left(t_{0}, x_{0}\right) \leqslant P^{*}\left(u^{*}, \nabla \varphi, \nabla^{2} \varphi\right)$

at any local minimizer $\left(t_{0}, x_{0}\right) \in(0, T) \times \mathbb{R}^{n}$ of the difference $u^{*}-\varphi$. 
(B) Similarly, we say that $u$ is a viscosity supersolution of $u_{t}+P\left(t, x, u, \nabla u, \nabla^{2} u\right)=0$ in $(0, T) \times \mathbb{R}^{n}$ if for any $\varphi \in C^{2}\left((0, T) \times \mathbb{R}^{n}\right)$

$\varphi_{t}\left(t_{0}, x_{0}\right) \geqslant P_{*}\left(u_{*}, \nabla \varphi, \nabla^{2} \varphi\right)$

at any local minimizer $\left(t_{0}, x_{0}\right) \in(0, T) \times \mathbb{R}^{n}$ of the difference $u_{*}-\varphi$.

(C) Finally, $u$ is a viscosity solution of $u_{t}+$ $P\left(t, x, u, \nabla u, \nabla^{2} u\right)=0$ in $(0, T) \times \mathbb{R}^{n}$ if it is both a viscosity subsolution and a viscosity supersolution.

Definition 3. [Crandall et al., 1992]. Let $u$ and $P$ be as in Definition 1. The function $P$ is said to be geometric if it satisfies $P(x, \lambda p, \lambda X+\mu p \otimes p)=\lambda P(x, p, X), \forall \lambda>0, \mu \in \mathbb{R}$, where $x, p \in \mathbb{R}^{n}, X \in S^{n \times n}$ and $\otimes$ denotes the tensor product of vectors in $\mathbb{R}^{n}$.

Theorem 1. [Theorem 6.8 (Chen et al., 1991)]. Let $P(t, x, p, X)$ continuous, degenerate elliptic and geometric in $(0, T] \times \mathbb{R}^{n} \times\left(\mathbb{R}^{n} \backslash\{0\}\right) \times S^{n \times n}$. We also suppose that $P$ satisfies

$$
\begin{aligned}
& -\infty<P_{*}(t, x, 0, O)=P^{*}(t, x, 0, O)<\infty, \\
& \left\{\begin{array}{l}
P_{*}(t, x, p,-I) \leqslant c_{-}(|p|), \\
P^{*}(t, x, p, I) \geqslant-c_{+}(|p|)
\end{array}\right.
\end{aligned}
$$

for some $c_{ \pm} \in C^{l}[0, \infty)$ and $c_{ \pm} \geqslant c_{0}>0$ with some constant $c_{0}$. Then for any uniformly continuous function $u_{0}$, there is a unique, uniformly continuous viscosity solution $u$ in $[0, T] \times \mathbb{R}^{n}$ of the initial value problem for the geometric parabolic equation:

$$
\left\{\begin{array}{l}
u_{t}+P\left(t, x, \nabla u, \nabla^{2} u\right)=0 \quad \text { in }(0, T] \times \mathbb{R}^{n} \\
u(0, x)=u_{0}(x) \text { in } \mathbb{R}^{n} .
\end{array}\right.
$$

Theorem 2. Our initial value partial differential equation, defined in (A.1) and (A.2), has a unique, uniformly continuous viscosity solution $\phi$ in $[0, T] \times \mathbb{R}^{n}$.

Proof. (A) First, we check if $G$ in (A.2) is continuous, degenerate elliptic and geometric in $(0, T] \times \mathbb{R}^{3} \times$ $\left(\mathbb{R}^{3} \backslash\{0\}\right) \times S^{3 \times 3}$.

- The front propagation speed, (9), depends on the tensor diffusion at points $x$ and $x-p N$ with $p=1$ and 2 and the normal vector $N=\frac{\nabla \phi}{\nabla \phi \mid}$ to the surface, so $F=F\left(x, \frac{\nabla \phi}{|\nabla \phi|}\right)$. Then, we suppose that $F$ is continuous in $\mathbb{R}^{3} \times\left(\mathbb{R}^{3} \backslash\{0\}\right)$ and since Heaviside function $H_{T}$ is $C^{2}$ then $H_{T}(G)$ is continuous.

Let $\lambda_{1}$ and $\lambda_{2}$ be the principal curvatures of level sets of $\phi$ in $\mathbb{R}^{3}$. If we order the principal curvatures in the increasing order, i.e. $\lambda_{1} \leqslant \lambda_{2}$, the minimal principal curvature of level sets of $\phi$ in $\mathbb{R}^{3}$ is then $\kappa=\min \left\{\lambda_{1}(J), \lambda_{2}(J)\right\}=\lambda_{1}(J)$,

with

$J=\frac{P_{\nabla \phi} \nabla^{2} \phi P_{\nabla \phi}}{|\nabla \phi|}$,

where $P_{p}$ is a projection operator onto the normal space to vector $p: P_{p}=I-\frac{p \otimes p}{|p|^{2}}$. According to Ambrosio and Soner (1996), the ${ }^{|p|^{2}}$ minimal principal curvature $\kappa=\kappa\left(x, \nabla \phi, \nabla^{2} \phi\right)$ is continuous in $\mathbb{R}^{3} \times\left(\mathbb{R}^{3} \backslash\{0\}\right) \times S^{3 \times 3}$ and so is the whole function $G\left(x, \nabla \phi, \nabla^{2} \phi\right)$ in $\mathbb{R}^{3} \times\left(\mathbb{R}^{3} \backslash\{0\}\right) \times S^{3 \times 3}$.

- We have to show that Condition 2 of Definition 1 is satisfied to prove that $G$ is degenerate elliptic. Since $F$ does not depend on $\nabla^{2} \phi$ the degenerate ellipticity condition is verified if

$$
\begin{gathered}
-\lambda_{1}(J(p, X)) \leqslant-\lambda_{1}(J(p, Y)) \text { with } X \geqslant Y, X, Y \in S^{3 \times 3}, \\
p \in \mathbb{R}^{3} \backslash\{0\} \text { and } J(p, X)=\frac{P_{p} X P_{p}}{|p|} .
\end{gathered}
$$

Eigenvalues of a matrix $X$ are defined in (Ambrosio and Soner, 1996) by the formula

$\lambda_{i}(X)=\max \left\{\min _{v \in E} \frac{X v \cdot v}{|v|^{2}}: E \subset H, \operatorname{codim}(E) \leqslant i-1\right\}$.

This means that (A.10) holds for $X \geqslant Y$ and that $G$ is degenerate elliptic.

- Definition 3 has to be satisfied to prove that $G$ is of geometric type, i.e.,

$$
\begin{aligned}
G(\lambda p, \lambda X+\mu p \otimes p)= & \lambda G(p, X), \forall \lambda>0, \mu \in \mathbb{R}, \\
& p \in \mathbb{R}^{3} \backslash\{0\} \text { and } X, Y \in S^{3 \times 3} .
\end{aligned}
$$

For the first term of $G$, we have

$H_{\mathrm{T}}\left(F\left(\frac{\lambda p}{|\lambda p|}\right)\right)|\lambda p|=\lambda H_{\mathrm{T}}(F)|p|$.

For the second term, using $P_{\lambda p}=P_{p}$ and $P_{p}(p$ $\otimes p)=0$, we have

$\alpha \lambda_{1}\left(\frac{P_{\lambda p}(\lambda X+\mu p \otimes p) P_{\lambda p}}{|\lambda p|}\right)|\lambda p|=\lambda \alpha \lambda_{1}|p|$.

This implies that $G$ is geometric.

(B) Second, we verify the conditions in (A.5) and (A.6). - When $\nabla \phi=0$, we follow Ambrosio and Soner (1996) since we take the same u.s.c. and 1.s.c. envelopes for the principal minimal curvature $\lambda_{1}$ :

$$
\left\{\begin{array}{l}
\lambda_{1}^{*}(0, X)=\max \left\{\lambda_{1}(v, X):|v|=1\right\}, \\
\lambda_{1 *}(0, X)=\min \left\{\lambda_{1}(v, X):|v|=1\right\} .
\end{array}\right.
$$

And we choose for $F$ independent of $X$ : 


$$
\left\{\begin{array}{l}
F^{*}(0)=\max \{F(v):|v|=1\}, \\
F_{*}(0)=\min \{F(v):|v|=1\} .
\end{array}\right.
$$

According to the form of (A.2), we have $G^{*}(0, O)=$ $G_{*}(0, O)=0$ at any $(t, x) \in(0, T] \times \mathbb{R}^{3}$ and so the condition in (A.5) is satisfied.

- Finally, $G$ is geometric and continuous for $p \in \mathbb{R}^{3} \backslash\{0\}, G$ satisfies the condition in (A.6) by taking

$c_{ \pm}(\rho)=\rho \sup _{|p|=1}\left|H_{\mathrm{T}}(F(p))\right|+\sup _{|p|=1} \alpha \lambda_{1}\left(P_{p}\right)$,

using $P_{p} P_{p}=P_{p}$.

(C) Theorem 1 can be applied to our geometric parabolic equation (A.1) since it satisfies all required conditions. Our PDE has a unique, uniformly continuous viscosity solution $\phi$ in $[0, T] \times \mathbb{R}^{3}$ for any initial uniformly continuous function $\phi_{0}$ in $\mathbb{R}^{3}$.

Theorem 3. [Theorem 7.1 (Chen et al., 1991)]. Suppose that $P, u$ and $u_{0}$ are as in Theorem 1. Let $S(t)$ and $D(t)$ be defined by

$S(t)=\left\{x \in \mathbb{R}^{n} ; u(t, x)=0\right\}$,

$D(t)=\left\{x \in \mathbb{R}^{n} ; u(t, x)>0\right\}$.

The evolution family $(S(t), D(t))$ for $t \geqslant 0$ is uniquely determined by $(S(0), D(0))$ and is independent of $u_{0}$. $(S(t), D(t))$ is called a solution family of (A.7) with initial data $(S(0), D(0))$.

We apply this theorem to our problem to say that the evolution of the active surface $S$ embedded as the zero level set of $\phi$ is independent with respect to the embedding solution $\phi_{0}$ which zero level set represents the initial active surface $S_{0}$.

\section{References}

Adalsteinsson, D., Sethian, J., 1995. A fast level set method for propagating interfaces. J. Comput. Phys. 118, 269-277.

Alexander, D., Gee, J., Bajcsy, R., 1999. Similarity measures for matching diffusion tensor images. In: Proc. BMCV'99.

Ambrosio, L., Soner, H., 1996. Level set approach to mean curvature flow in arbitrary codimension. J. Differ. Geom. 43, 693-737.

Basser, P., Mattiello, J., Le Bihan, D., 1994. MR diffusion tensor spectography and imaging. Biophys. J. 66, 259-267.

Basser, P., Pajevic, S., 2000. Statistical artefacts in diffusion tensor MRI (DT-MRI) caused by background noise. Magn. Reson. Med. 44 (1), 41-50.

Basser, P., Pajevic, S., Pierpaoli, C., Duda, J., Aldroubi, A., 2000. In vivo fiber tractography using DT-MRI data. Magn. Reson. Med. 44 (4), 625-632.

Basser, P., Pierpaoli, C., 1998. A simplified method to measure the diffusion tensor from seven MR images. Magn. Reson. Med. 39, 928-934.

Batchelor, P., Hill, F., Calamante, F., Atkinson, D., 2001. Study of connectivity in the brain using the full diffusion tensor from MRI. In: IPMI, pp. 121-133.

Beaulieu, C., 2002. The basis of anisotropic water diffusion in the nervous system - a technical review. NMR Biomed. 15 (7-8), 435455 .
Campbell, J.S.W., Siddiqi, K., Vemuri, B.C., Pike, G.B., 2002. A geometric flow for white matter fibre tract reconstruction. In: Int. Symp. Biomed. Imag., http://www.cim.mcgill.ca/siddiqi/conference. html.

Caselles, V., Kimmel, R., Sapiro, G., 1997. Geodesic active contours. Int. J. Comput. Vis. 22, 61-79.

Chefd'hotel, C., Tschumperl'e, D., Deriche, R., Faugeras, O., 2002. Constrained flows of matrix-valued functions: application to diffusion tensor regularization. In: ECCV (1), pp. 251-265.

Chen, Y., Giga, Y., Goto, S., 1991. Uniqueness and existence of viscosity solutions of generalized mean curvature flow equations. J. Differ. Geom. 33, 749-786.

Clarke, S., Kraftsik, R., van der Loos, H., Innocenti, G., 1989. Forms and measures of adult and developing human corpus callosum: is there sexual dimorphism. J. Comp. Neurol. 280, 213-230.

Conturo, T., Lori, N., Cull, T., Akbudak, E., Snyder, A., Shimony, J., McKinstry, R., Burton, H., Raichle, M., 1999. Tracking neuronal fiber pathways in the living human brain. Proc. Natl. Acad. Sci. USA 96 (18), 10422-10427.

Coulon, O., Alexander, D., Arridge, S., 2004. Diffusion tensor magnetic resonance image regularisation. Med. Image Anal. 8 (1), 47-67.

Crandall, M., Ishi, H., Lions, P., 1992. Users' guide to viscosity solutions of second order partial differential equations. Bull. Amer. Math. Soc. 27 (1), 1-69.

Feddern, C., Weickert, J., Burgeth, B., 2003. Level-set methods for tensor valued images. In: Proceedings of the Second IEEE Workshop on Variational, Geometric and Level Set Methods in Computer Vision, pp. 65-72.

Filippi, M., Cercignani, M., Inglese, M., Horsfield, M., Comi, G., 2001. Diffusion tensor magnetic resonance imaging in multiple sclerosis. Neurology 56 (3), 304-311.

Foong, J., Maier, M., Clark, C., Barker, G., Miller, D., Ron, M., 2000. Neuropathological abnormalities of the corpus callosum in schizophrenia: a diffusion tensor imaging study. J. Neurol. Neurosurg. Psychiatry 68 (2), 242-244.

Gudbjartsson, H., Patz, S., 1995. The Rician distribution of noisy MRI data. Magn. Reson. Med. 34, 910-914.

Hagmann, P., Thiran, J., Jonasson, L., Vandergheynst, P., Clarke, S., Meuli, R., 2003. Dti mapping of human brain connectivity: Statistical fibre tracking and virtual dissection. NeuroImage 19 (3), 545-554.

Henkelman, R., 1985. Measurement of signal intensities in the presence of noise in MR images. Med. Phys. 12, 232-233.

Klingberg, T., Hedehus, M., Temple, E., Salz, T., Gabrieli, J., Moseley, M., Poldrack, R., 2000. Microstructure of temporoparietal white matter as a basis for reading ability: evidence from diffusion tensor magnetic resonance imaging. Neuron 25 (2), 493 500

Lim, K., Hedehus, M., Moseley, M., de Crespigny, A., Sullivan, E., Pfeffer- baum, A., 1999. Compromised white matter tract integrity in schizophrenia inferred from diffusion tensor imaging. Arch. Gen. Psychiatry 56 (4), 367-374.

Lim, K., Helpern, J., 2002. Neuropsychiatric applications of DTI - a review. NMR Biomed. 15, 587-593.

Lorigo, L., Faugeras, O., Grimson, W., Keriven, R., Kikinis, R., Westin, C., 1999. Co-dimension 2 geodesic active contours for MRA segmentation. In: IPMI 1999, pp. 126-139.

Maldjian, J., Grossman, R., 2001. Future applications of DWI in MS. J. Neurol. Sci. 186 (Suppl. 1), S55-S57.

O'Donnell, L., S., H., C.F., W., 2002. New approaches to estimation of white matter connectivity in diffusion tensor MRI: elliptic pdes and geodesics in a tensor-warped space. In: MICCAI2002.

Osher, S., Sethian, J., 1988. Fronts propagating with curvaturedependent speed: algorithms based on Hamilton-Jacobi formulations. J. Comput. Phys. 79, 12-49, http://www.citeseer.nj.nec.com/ osher88fronts.html. 
Pajevic, S., Pierpaoli, C., 1999. Color schemes to represent the orientation of anisotropic tissues from diffusion tensor data: application to white matter fiber tract mapping in the human brain. Magn. Reson. Med. 42 (3), 526-540.

Paragios, N., Deriche, R., Oct. 1999. Coupled geodesic active regions for image segmentation. Technical Report 3783, INRIA.

Parker, G., Wheeler-Kingshott, C., Barker, G., 2001. Distributed anatomical brain connectivity derived from diffusion tensor imaging. In: IPMI 2001, pp. 106-120.

Pettey, D., Gee, J., 2002. Sexual dimorphism in the corpus callosum: a characteristic of local size variations and a classification driven approach to morphometry. NeuroImage $17,1504-1511$.

Pfefferbaum, A., Sullivan, E., 2002. Microstructural but not macrostructural disruption of white matter in women with chronic alcholism. NeuroImage 15, 708-718.

Rousson, M., Lenglet, C., Deriche, R., 2004. Level set and region based surface propagation for diffusion tensor MRI segmentation. In: CVAMIA and MMBIA.

Sethian, J.A., 1999. Level Set Methods and Fast Marching Methods: Evolving Interfaces in Computational Geometry, Fluid Mechanics, Computer Vision, and Materials Science. Cambridge University Press, 1999.
Sussman, M., Fatemi, E., Smereka, P., Osher, S., 1998. An improved level set method for incompressible two-phase flows. Comput. Fluids 27 (5-6), 663-680.

Tench, C., Morgan, P., Wilson, M., Blumhardt, L., 2002. White matter mapping using diffusion tensor MRI. Magn. Reson. Med. 47 (5), 967-972.

Tournier, J., Calamante, F., King, M., Gadian, D., Connelly, A., 2002. Limitations and requirements of diffusion tensor fiber tracking: an assessment using simulations. Magn. Reson. Med. 47 (4), 701-708.

Tschumperle, D., Deriche, R., 2001. Diffusion tensor regularization with constraints preservation. In: CVPR'2001, Computer Vision and Pattern Recognition.

Vemuri, B., Chen, Y., R.M., McGraw, T., Wang, Z., Mareci, T., 2001. Fiber tract mapping from diffusion tensor MRI. In: Proceedings of the IEEE Workshop on Variational and Level Set Methods in Computer Vision, pp. 81-88.

Wang, Z., Vemuri, B., 2004. Tensor field segmentation using region based active contour model. In: ECCV 2004, LNCS 3024. Springer, Berlin, pp. 304-315.

Zhukov, L., Museth, K., Breen, D., Whitaker, R., Barr, A., 2003. Level set modelling and segmentation of DT-MRI brain data. J. Electron. Imag. 12 (1), 125-133. 